Pacific

Journal of

Mathematics

\title{
MAXIMAL ESTIMATES FOR SCHRÖDINGER EQUATIONS WITH INVERSE-SQUARE POTENTIAL
}

Changxing MiaO, Junyong Zhang AND JiQIANG Zheng 


\title{
MAXIMAL ESTIMATES FOR SCHRÖDINGER EQUATIONS WITH INVERSE-SQUARE POTENTIAL
}

\author{
ChangXing MiaO, Junyong Zhang And JiQiang Zheng
}

\begin{abstract}
We consider maximal estimates for the solution to an initial value problem of the linear Schrödinger equation with a singular potential. We show a result about the pointwise convergence of solutions to this special variable coefficient Schrödinger equation with initial data $u_{0} \in H^{s}\left(\mathbb{R}^{n}\right)$ for $s>\frac{1}{2}$ or radial initial data $u_{0} \in H^{s}\left(\mathbb{R}^{n}\right)$ for $s \geqslant \frac{1}{4}$ and that the solution does not converge when $s<\frac{1}{4}$.
\end{abstract}

\section{Introduction and statement of main result}

We study the maximal estimates for the solution to an initial value problem of the linear Schrödinger equation with an inverse square potential. More precisely, we consider the Schrödinger equation

$$
\left\{\begin{array}{l}
i \partial_{t} u-\Delta u+\frac{a}{|x|^{2}} u=0 \quad(t, x) \in \mathbb{R} \times \mathbb{R}^{n} \backslash\{0\}, a>-(n-2)^{2} / 4, \\
u(x, 0)=u_{0}(x) .
\end{array}\right.
$$

The scale-covariance elliptic operator $P_{a}:=-\Delta+a /|x|^{2}$ appearing in (1-1) plays a key role in many problems of physics and geometry. The heat and wave flows for the elliptic operator $P_{a}$ have been studied in the theory of combustion (see [Vazquez and Zuazua 2000]) and in wave propagation on conic manifolds (see [Cheeger and Taylor 1982]). The Schrödinger equation (1-1) arises in the study of quantum mechanics [Kalf et al. 1975]. There has been a lot of interest in developing Strichartz estimates both for the Schrödinger and wave equations with the inverse square potential; we refer the reader to [Burq et al. 2003; 2004; Planchon et al. 2003a; 2003b; Miao et al. 2013]. However, as far as we know, there are few results about the maximal estimates associated with the operator $P_{a}$, which arises in the study of pointwise convergence problem for the Schrödinger and wave equations with the inverse square potential. In this paper, we aim to address some maximal estimates in the special settings associated with the operator $P_{a}$. As a

MSC2010: 35B65, 35Q55, 47J35.

Keywords: inverse square potential, maximal estimate, spherical harmonics. 
direct consequence, we obtain the pointwise convergence result for $u_{0} \in H^{s}\left(\mathbb{R}^{n}\right)$ with $s>\frac{1}{2}$.

In the case of the free Schrödinger equation without potential, i.e., $a=0$, there is a large amount of literature on developing maximal estimates for its solution, which can be formally written as

$$
u(t, x)=e^{i t \Delta} u_{0}(x)=\int_{\mathbb{R}^{n}} e^{2 \pi i\left(x \cdot \xi-t|\xi|^{2}\right)} \hat{u}_{0}(\xi) \mathrm{d} \xi .
$$

When $n=1$, Carleson [1980] proved that the convergence result holds in the sense that $\lim _{t \rightarrow 0} u(t)=u_{0}$ for a.e. $x$ when $u_{0} \in H^{s}(\mathbb{R})$ with $s \geqslant \frac{1}{4}$. Dahlberg and Kenig [1982] showed that the result is sharp in the sense that the solution does not converge when $s<\frac{1}{4}$. When $n \geqslant 2$, Sjölin [1987] and Vega [1988] independently proved convergence results when $u_{0} \in H^{s}\left(\mathbb{R}^{n}\right)$ with $s>\frac{1}{2}$. It follows from the construction of [Dahlberg and Kenig 1982; Vega 1988] that the solution does not converge when $s<\frac{1}{4}$. When $n=2$, Bourgain [1995] showed that there is a certain $s<\frac{1}{2}$ such that the convergence result holds, and this result was improved by Moyua, Vargas and Vega [Moyua et al. 1996]. Having shown the bilinear restriction estimates for paraboloids, Tao and Vargas [2000] and Tao [2003] showed convergence for $s>\frac{15}{32}$ and $s>\frac{2}{5}$ respectively. This was improved further to $s>\frac{3}{8}$ in [Lee 2006; Shao 2010]. Very recently, Bourgain [2013] made some progress in high dimension $n \geqslant 2$ to show that the convergence result holds for $s>\frac{1}{2}-\frac{1}{4 n}$ when $n \geqslant 1$ and that the convergence result needs $s \geqslant(n-2) /(2 n)$ when $n \geqslant 5$.

In the situation when $a \neq 0,(1-1)$ can be viewed as a special Schrödinger equation with variable singular coefficients. The potential prevents us from using the Fourier transform to give the expression of the solution. With the motivation of regarding the potential term as a perturbation on angular direction in [Burq et al. 2003; Planchon et al. 2003b; Miao et al. 2013], we express the solution by using the Hankel transform of radial functions and spherical harmonics. Instead of the Fourier transform, we utilize the Hankel transform and modify the argument of [Vega 1988] to show that the pointwise convergence result holds when the initial data $u_{0} \in H^{s}\left(\mathbb{R}^{n}\right)$ for $s>\frac{1}{2}$, or when radial initial data $u_{0} \in H^{s}\left(\mathbb{R}^{n}\right)$ for $s \geqslant \frac{1}{4}$, and that the solution does not converge when $s<\frac{1}{4}$.

Let $u$ be the solution to (1-1); we define the maximal function by

$$
u^{*}(x)=\sup _{|t|>0}|u(x, t)| .
$$

Our main theorems are the following.

Theorem 1.1. Let $\beta>1, n \geqslant 2$ and $s>\frac{1}{2}$. Then

$$
\int_{\mathbb{R}^{n}}\left|u^{*}(x)\right|^{2} \frac{\mathrm{d} x}{(1+|x|)^{\beta}} \leqslant C\left\|u_{0}\right\|_{H^{s}\left(\mathbb{R}^{n}\right)}^{2}
$$


As a direct consequence of Theorem 1.1, we have:

Corollary 1.1. Let $u_{0} \in H^{s}\left(\mathbb{R}^{n}\right)$ with $s>\frac{1}{2}$ and $n \geqslant 2$. Then

$$
\lim _{t \rightarrow 0} u(t, x)=u_{0}(x), \quad \text { a.e. } x \in \mathbb{R}^{n} .
$$

Theorem 1.2. Let $B^{n}$ be the open unit ball in $\mathbb{R}^{n}$. Assume that there exists a constant $C$ independent of $u_{0}$ such that

$$
\int_{B^{n}}\left|u^{*}(x)\right|^{2} \mathrm{~d} x \leqslant C\left\|u_{0}\right\|_{H^{s}\left(\mathbb{R}^{n}\right)}^{2} \quad \text { for all } u_{0}(x) \in H^{s}\left(\mathbb{R}^{n}\right) .
$$

Then $s \geqslant \frac{1}{4}$.

With this in mind, Theorem 1.1 is far from being sharp. Assuming that the initial data possesses additional angular regularity, we have:

Theorem 1.3. Let $B^{n}$ be the open unit ball in $\mathbb{R}^{n}$ and $\epsilon>0$. There exists a constant $C$ independent of $u_{0}$ such that

$$
\int_{B^{n}}\left|u^{*}(x)\right|^{2} \mathrm{~d} x \leqslant C\left\|u_{0}\right\|_{H_{r}^{\frac{1}{4}} H_{\theta}^{2}}^{\frac{n-1}{2}+\epsilon},
$$

where for $s, s^{\prime} \geqslant 0$,

$H_{r}^{s} H_{\theta}^{s^{\prime}}=\left\{g:\|g\|_{H_{r}^{s} H_{\theta}^{s^{\prime}}}:=\left\|\left(1-\Delta_{\theta}\right)^{\frac{s^{\prime}}{2}}\left((1-\Delta)^{\frac{s}{2}} g\right)\right\|_{L_{r n-1}^{2}\left(\mathbb{R}^{+} ; L_{\theta}^{2}\left(\mathbb{S}^{n-1}\right)\right)}<\infty\right\}$.

Here $\Delta_{\theta}$ denotes the Laplace-Beltrami operator on $\mathbb{S}^{n-1}$.

Remark 1.1. i) This result implies that the pointwise convergence of solutions to (1-1) holds for radial initial data $u_{0} \in H^{s}\left(\mathbb{R}^{n}\right)$ with $s \geqslant \frac{1}{4}$.

ii) This result is an analogue of [Cho et al. 2006, Theorem 1.1]. We remark that the parameter $\epsilon$ there should be corrected to $\epsilon>\frac{1}{2}$ rather than $\epsilon>0$. Thus, we generalize and improve the result of Cho et al. by making use of a finer result proved in [Gigante and Soria 2008].

Now we introduce some notation. We use $A \lesssim B$ to denote the statement that $A \leqslant C B$ for some large constant $C$ which may vary from line to line and depend on various parameters; and similarly use $A \ll B$ to denote the statement $A \leqslant C^{-1} B$. We employ $A \sim B$ to denote the statement that $A \lesssim B \lesssim A$. If the constant $C$ depends on a special parameter other than the above, we shall denote it explicitly by subscripts. We briefly write $A+\epsilon$ as $A+$ or $A-\epsilon$ as $A-$ for $0<\epsilon \ll 1$. Throughout this paper, pairs of conjugate indices are written as $p, p^{\prime}$, where $1 / p+1 / p^{\prime}=1$ with $1 \leqslant p \leqslant \infty$.

This paper is organized as follows. In Section 2, we mainly revisit the properties of the Bessel functions and the Hankel transform associated with $-\Delta+a /|x|^{2}$. Section 3 is devoted to the proofs of the theorems. 


\section{Preliminaries}

We list some results about the Hankel transform and the Bessel functions and then show a characterization of the Sobolev norm in the Hankel transform version.

We begin by recalling the expansion formula with respect to spherical harmonics. For details, we refer to [Stein and Weiss 1971]. For the sake of convenience, let

$$
\xi=\rho \omega \quad \text { and } \quad x=r \theta \quad \text { with } \omega, \theta \in \mathbb{S}^{n-1} .
$$

For any $g \in L^{2}\left(\mathbb{R}^{n}\right)$, the expansion formula with respect to the spherical harmonics yields

$$
g(x)=\sum_{k=0}^{\infty} \sum_{\ell=1}^{d(k)} a_{k, \ell}(r) Y_{k, \ell}(\theta)
$$

where

$$
\left\{Y_{k, 1}, \ldots, Y_{k, d(k)}\right\}
$$

is the orthogonal basis of the space of spherical harmonics of degree $k$ on $\mathbb{S}^{n-1}$, called $\mathscr{H}^{k}$, having dimension

$$
d(k)=\frac{2 k+n-2}{k} C_{n+k-3}^{k-1} \simeq\langle k\rangle^{n-2} .
$$

We remark that for $n=2$, the dimension of $\mathscr{H}^{k}$ is independent of $k$. Obviously, we have the orthogonal decomposition

$$
L^{2}\left(\mathbb{S}^{n-1}\right)=\bigoplus_{k=0}^{\infty} \mathscr{H}^{k}
$$

By orthogonality, it gives

$$
\|g(x)\|_{L_{\theta}^{2}\left(\mathbb{S}^{n-1}\right)}=\left\|a_{k, \ell}(r)\right\|_{\ell_{k, \ell}^{2}} .
$$

From $-\Delta_{\theta} Y_{k, \ell}(\theta)=k(k+n-2) Y_{k, \ell}(\theta)$, the fractional power of $1-\Delta_{\theta}$ can be written explicitly [Machihara et al. 2005] as

$$
\left(1-\Delta_{\theta}\right)^{\frac{s}{2}} g(x)=\sum_{k=0}^{\infty} \sum_{\ell=1}^{d(k)}(1+k(k+n-2))^{\frac{s}{2}} a_{k, \ell}(r) Y_{k, \ell}(\theta) .
$$

We will need the Fourier transform of $a_{k, \ell}(r) Y_{k, \ell}(\theta)$. Theorem 3.10 of [Stein and Weiss 1971] asserts the Hankel transform formula

$$
\hat{g}(\rho \omega) \sim \sum_{k=0}^{\infty} \sum_{\ell=1}^{d(k)} i^{k} Y_{k, \ell}(\omega) \rho^{-\frac{n-2}{2}} \int_{0}^{\infty} J_{k+\frac{n-2}{2}}(2 \pi r \rho) a_{k, \ell}(r) r^{\frac{n}{2}} \mathrm{~d} r .
$$


Here the Bessel function $J_{k}(r)$ of order $k$ is defined by the integral

$$
J_{k}(r)=\frac{\left(\frac{r}{2}\right)^{k}}{\Gamma\left(k+\frac{1}{2}\right) \Gamma\left(\frac{1}{2}\right)} \int_{-1}^{1} e^{i s r}\left(1-s^{2}\right)^{\frac{2 k-1}{2}} d s \quad \text { with } k>-\frac{1}{2} \text { and } r>0 .
$$

A simple computation gives the rough estimates

$$
\left|J_{k}(r)\right| \leqslant \frac{C r^{k}}{2^{k} \Gamma\left(k+\frac{1}{2}\right) \Gamma\left(\frac{1}{2}\right)}\left(1+\frac{1}{k+\frac{1}{2}}\right),
$$

where $C$ is an absolute constant. This estimate will be mainly used when $r \lesssim 1$. Another well-known asymptotic expansion for the Bessel function is

$$
J_{k}(r)=r^{-\frac{1}{2}} \sqrt{\frac{2}{\pi}} \cos \left(r-\frac{k \pi}{2}-\frac{\pi}{4}\right)+O_{k}\left(r^{-\frac{3}{2}}\right) \quad \text { as } r \rightarrow \infty,
$$

but with a constant depending on $k$ (see [Stein and Weiss 1971]). As pointed out in [Stein 1993], if one seeks a uniform bound for large $r$ and $k$, the best one can do is $\left|J_{k}(r)\right| \leqslant C r^{-\frac{1}{3}}$. One will find that this decay doesn't lead to the desired result.

We now recall the properties of Bessel function $J_{k}(r)$ in [Stein 1993; Stempak 2000].

Lemma 2.1 (asymptotics of the Bessel function). Assume that $k \in \mathbb{N}$ and $k \gg 1$. Let $J_{k}(r)$ be the Bessel function of order $k$ defined as above. There exist a large constant $C$ and small constant $c$ independent of $k$ and $r$ satisfying these conditions:

- When $r \leqslant \frac{k}{2}$,

$$
\left|J_{k}(r)\right| \leqslant C e^{-c(k+r)} .
$$

- When $\frac{k}{2} \leqslant r \leqslant 2 k$,

$$
\left|J_{k}(r)\right| \leqslant C k^{-\frac{1}{3}}\left(k^{-\frac{1}{3}}|r-k|+1\right)^{-\frac{1}{4}} .
$$

- When $r \geqslant 2 k$,

$$
J_{k}(r)=r^{-\frac{1}{2}} \sum_{ \pm} a_{ \pm}(r, k) e^{ \pm i r}+E(r, k)
$$

where $\left|a_{ \pm}(r, k)\right| \leqslant C$ and $|E(r, k)| \leqslant C r^{-1}$.

As a consequence of Lemma 2.1, we have:

Lemma 2.2. Let $R \gg 1$. There exists a constant $C$ independent of $k, R$ such that

$$
\int_{R}^{2 R}\left|J_{k}(r)\right|^{2} \mathrm{~d} r \leqslant C
$$


Proof. To prove (2-10), we write

$$
\int_{R}^{2 R}\left|J_{k}(r)\right|^{2} \mathrm{~d} r=\int_{I_{1}}\left|J_{k}(r)\right|^{2} \mathrm{~d} r+\int_{I_{2}}\left|J_{k}(r)\right|^{2} \mathrm{~d} r+\int_{I_{3}}\left|J_{k}(r)\right|^{2} \mathrm{~d} r
$$

where $I_{1}=[R, 2 R] \cap\left[0, \frac{k}{2}\right], I_{2}=[R, 2 R] \cap\left[\frac{k}{2}, 2 k\right]$ and $I_{3}=[R, 2 R] \cap[2 k, \infty]$. By (2-7) and (2-9), we have

$$
\int_{I_{1}}\left|J_{k}(r)\right|^{2} \mathrm{~d} r \leqslant C \int_{I_{1}} e^{-c r} \mathrm{~d} r \leqslant C e^{-c R},
$$

and

$$
\int_{I_{3}}\left|J_{k}(r)\right|^{2} \mathrm{~d} r \leqslant C
$$

On the other hand, one has by (2-8)

$$
\int_{\left[\frac{k}{2}, 2 k\right]}\left|J_{k}(r)\right|^{2} \mathrm{~d} r \leqslant C \int_{\left[\frac{k}{2}, 2 k\right]} k^{-\frac{2}{3}}\left(1+k^{-\frac{1}{3}}|r-k|\right)^{-\frac{1}{2}} \mathrm{~d} r \leqslant C .
$$

Observing that $[R, 2 R] \cap\left[\frac{k}{2}, 2 k\right]=\varnothing$ unless $R \sim k$, we obtain

$$
\int_{I_{2}}\left|J_{k}(r)\right|^{2} \mathrm{~d} r \leqslant C
$$

This together with (2-11) and (2-12) yields (2-10).

For simplicity, we define

(2-14) $\mu(k)=\frac{n-2}{2}+k \quad$ and $\quad v(k)=\sqrt{\mu^{2}(k)+a} \quad$ with $a>-(n-2)^{2} / 4$.

We sometime write $v$ instead of $v(k)$. Let $f$ be a Schwartz function defined on $\mathbb{R}^{n}$. We define the Hankel transform of order $v$ by

$$
\left(\mathscr{H}_{\nu} f\right)(\xi)=\int_{0}^{\infty}(r \rho)^{-\frac{n-2}{2}} J_{v}(r \rho) f(r \omega) r^{n-1} \mathrm{~d} r,
$$

where $\rho=|\xi|, \omega=\xi /|\xi|$ and $J_{v}$ is the Bessel function of order $v$. In particular, if the function $f$ is radial, then we have

$$
\left(\mathscr{H}_{\nu} f\right)(\rho)=\int_{0}^{\infty}(r \rho)^{-\frac{n-2}{2}} J_{v}(r \rho) f(r) r^{n-1} \mathrm{~d} r .
$$

If $f(x)=\sum_{k=0}^{\infty} \sum_{\ell=1}^{d(k)} a_{k, \ell}(r) Y_{k, \ell}(\theta)$, it follows from (2-4) that

$$
\hat{f}(\xi)=\sum_{k=0}^{\infty} \sum_{\ell=1}^{d(k)} 2 \pi i^{k} Y_{k, \ell}(\omega)\left(\mathscr{H}_{\mu(k)} a_{k, \ell}\right)(\rho) .
$$


The following properties of the Hankel transform are obtained in [Burq et al. 2003; Planchon et al. 2003b].

Lemma 2.3. Let $\mathscr{H}_{v}$ be as above and set

$$
A_{v(k)}:=-\partial_{r}^{2}-\frac{n-1}{r} \partial_{r}+\left[v^{2}(k)-\left(\frac{n-2}{2}\right)^{2}\right] r^{-2} .
$$

(i) $\mathscr{H}_{v}=\mathscr{H}_{v}^{-1}$.

(ii) $\mathscr{H}_{v}$ is self-adjoint, i.e., $\mathscr{H}_{v}=\mathscr{H}_{v}^{*}$.

(iii) $\mathscr{H}_{\nu}$ is an $L^{2}$ isometry, i.e., $\left\|\mathscr{H}_{\nu} \phi\right\|_{L_{\xi}^{2}}=\|\phi\|_{L_{x}^{2}}$.

(iv) $\mathscr{H}_{\nu}\left(A_{\nu} \phi\right)(\xi)=|\xi|^{2}\left(\mathscr{H}_{\nu} \phi\right)(\xi)$, for $\phi \in L^{2}$.

We next recall an almost orthogonality inequality. Denote by $P_{j}$ and $\tilde{P}_{j}$ the usual dyadic frequency localization at $|\xi| \sim 2^{j}$ and the localization with respect to $\left(-\Delta+a /|x|^{2}\right)^{\frac{1}{2}}$. We define the projectors $M_{j j^{\prime}}=P_{j} \tilde{P}_{j^{\prime}}$ and $N_{j j^{\prime}}=\tilde{P}_{j} P_{j^{\prime}}$. More precisely, let $f$ be in the $k$-th harmonic subspace; then

$$
P_{j} f=\mathscr{H}_{\mu(k)} \beta_{j} \mathscr{H}_{\mu(k)} f \text { and } \tilde{P}_{j} f=\mathscr{H}_{v(k)} \beta_{j} \mathscr{H}_{v(k)} f,
$$

where $\beta_{j}(\xi)=\beta\left(2^{-j}|\xi|\right)$ with $\beta \in C_{0}^{\infty}\left(\mathbb{R}^{+}\right)$supported in $\left[\frac{1}{2}, 2\right]$.

Lemma 2.4 (almost orthogonality inequality [Burq et al. 2003]). Let $f \in L^{2}\left(\mathbb{R}^{n}\right)$. There exists a constant $C$ independent of $j, j^{\prime}$ such that

$$
\left\|M_{j j^{\prime}} f\right\|_{L^{2}\left(\mathbb{R}^{n}\right)},\left\|N_{j j^{\prime}} f\right\|_{L^{2}\left(\mathbb{R}^{n}\right)} \leqslant C 2^{-\epsilon\left|j-j^{\prime}\right|}\|f\|_{L^{2}\left(\mathbb{R}^{n}\right)},
$$

where $\epsilon<1+\min \left\{\frac{n-2}{2},\left(\frac{(n-2)^{2}}{4}+a\right)^{\frac{1}{2}}\right\}$.

As a consequence, we have:

Lemma 2.5. Let $f \in L^{2}\left(\mathbb{R}^{n}\right)$ be given by

$$
f(x)=\sum_{k=0}^{\infty} \sum_{\ell=1}^{d(k)} a_{k, \ell}(r) Y_{k, \ell}(\theta)
$$

Then for $0 \leqslant s<1+\min \left\{\frac{n-2}{2},\left(\frac{(n-2)^{2}}{4}+a\right)^{\frac{1}{2}}\right\}$ and $s^{\prime} \geqslant 0$,

$$
\sum_{k=0}^{\infty} \sum_{\ell=1}^{d(k)} \sum_{M \in 2^{\mathbb{Z}}} M^{2 s}(1+k)^{2 s^{\prime}}\left\|b_{k, \ell}(\rho) \chi\left(\frac{\rho}{M}\right) \rho^{\frac{n-1}{2}}\right\|_{L_{\rho}^{2}}^{2} \sim\|f\|_{\dot{H}_{r}^{s} H_{\theta}^{s^{\prime}}}^{2}
$$

where $b_{k, \ell}(\rho)=\left(\mathscr{H}_{\nu(k)} a_{k, \ell}\right)(\rho)$ and $\chi \in C_{0}^{\infty}\left(\mathbb{R}^{n}\right)$ such that supp $\chi \subset\left[\frac{1}{2}, 1\right]$.

Proof. Note that $-\Delta_{\theta} Y_{k, \ell}=k(k+n-2) Y_{k, \ell}$. By Lemma 2.3, we have 


$$
\begin{aligned}
\|f\|_{\dot{H}_{r}^{0} H_{\theta}^{s^{\prime}}}^{2} & \sim \sum_{k=0}^{\infty} \sum_{\ell=1}^{d(k)}(1+k)^{2 s^{\prime}}\left\|a_{k, \ell}(r)\right\|_{L_{r}^{2} n-1 \mathrm{~d} r}^{2}\left(\mathbb{R}^{+}\right) \\
& \sim \sum_{k=0}^{\infty} \sum_{\ell=1}^{d(k)}(1+k)^{2 s^{\prime}}\left\|b_{k, \ell}(\rho)\right\|_{L_{\theta}^{2}}^{2}{ }_{L^{n-1} \mathrm{~d} \rho}^{2}\left(\mathbb{R}^{+}\right)^{*}
\end{aligned}
$$

By (2-3), it suffices to show (2-19) with $s^{\prime}=0$. By Lemma 2.3, we have

$$
\begin{aligned}
\left\|b_{k, \ell}(\rho) \chi\left(\frac{\rho}{M}\right) \rho^{\frac{n-1}{2}}\right\|_{L_{\rho}^{2}} & =\left\|\chi\left(\frac{\rho}{M}\right) \mathscr{H}_{\nu}\left[Y_{k, l}(\theta) a_{k, \ell}(r)\right](\xi)\right\|_{L_{\xi}^{2}} \\
& =\left\|\mathscr{H}_{\nu}\left[\chi\left(\frac{\rho}{M}\right) \mathscr{H}_{\nu}\left(Y_{k, l}(\theta) a_{k, \ell}(r)\right)(\xi)\right]\right\|_{L_{x}^{2}}
\end{aligned}
$$

This yields, by letting $j=\log _{2} M$,

$$
\begin{aligned}
\left\|b_{k, \ell}(\rho) \chi\left(\frac{\rho}{M}\right) \rho^{\frac{n-1}{2}}\right\|_{L_{\rho}^{2}} & =\left\|\left[\mathscr{H}_{v} \chi\left(\frac{\rho}{M}\right) \mathscr{H}_{v}\right]\left(Y_{k, l}(\theta) a_{k, \ell}(r)\right)\right\|_{L_{x}^{2}} \\
& =\left\|\tilde{P}_{j}\left(Y_{k, l}(\theta) a_{k, \ell}(r)\right)\right\|_{L_{x}^{2}} .
\end{aligned}
$$

Let $g_{k, \ell}(x)=Y_{k, l}(\theta) a_{k, \ell}(r)$ and $\overline{P_{j^{\prime}}}=P_{j^{\prime}-1}+P_{j^{\prime}}+P_{j^{\prime}+1}$. We have by the triangle inequality and Lemma 2.4

$$
\begin{aligned}
\text { LHS of (2-19) } & =\sum_{k=0}^{\infty} \sum_{\ell=1}^{d(k)} \sum_{j \in \mathbb{Z}} 2^{2 s j}\left\|\tilde{P}_{j} g_{k, \ell}\right\|_{L_{x}^{2}}^{2} \\
& \lesssim \sum_{k=0}^{\infty} \sum_{\ell=1}^{d(k)} \sum_{j \in \mathbb{Z}} 2^{2 s j}\left(\sum_{j^{\prime}}\left\|\tilde{P}_{j} \overline{P_{j^{\prime}}} P_{j^{\prime}} g_{k, \ell}\right\|_{L_{x}^{2}}\right)^{2} \\
& \lesssim \sum_{k=0}^{\infty} \sum_{\ell=1}^{d(k)} \sum_{j \in \mathbb{Z}} 2^{2 s j}\left(\sum_{j^{\prime}} 2^{-\epsilon\left|j-j^{\prime}\right|}\left\|P_{j^{\prime}} g_{k, \ell}\right\|_{L_{x}^{2}}\right)^{2},
\end{aligned}
$$

where $s<\epsilon<1+\min \left\{\frac{n-2}{2},\left(\frac{(n-2)^{2}}{4}+a\right)^{\frac{1}{2}}\right\}$. Let $0<\epsilon_{1} \ll 1$ be such that $\epsilon_{2}:=\epsilon-\epsilon_{1}>s$; then the LHS of (2-19) is bounded above by

$$
\begin{gathered}
C \sum_{k=0}^{\infty} \sum_{\ell=1}^{d(k)} \sum_{j \in \mathbb{Z}} 2^{2 j s} \sum_{j^{\prime}} 2^{-2 \epsilon_{2}\left|j-j^{\prime}\right|}\left\|P_{j^{\prime}} g_{k, \ell}\right\|_{L^{2}\left(\mathbb{R}^{n}\right)}^{2} \sum_{j^{\prime}} 2^{-2 \epsilon_{1}\left|j-j^{\prime}\right|} \\
\leqslant C \sum_{k=0}^{\infty} \sum_{\ell=1}^{d(k)} \sum_{j^{\prime}} 2^{2 j^{\prime} s} \sum_{j \in \mathbb{Z}} 2^{2 j s} 2^{-2 \epsilon_{2}|j|}\left\|P_{j^{\prime}} g_{k, \ell}\right\|_{L^{2}\left(\mathbb{R}^{n}\right)}^{2} \\
\leqslant C \sum_{k=0}^{\infty} \sum_{\ell=1}^{d(k)} \sum_{j^{\prime}} 2^{2 j^{\prime} s}\left\|P_{j^{\prime}} g_{k, \ell}\right\|_{L^{2}\left(\mathbb{R}^{n}\right)}^{2}
\end{gathered}
$$


By the definition of $P_{j^{\prime}}$, Lemma 2.3 and (2-17), we have

$$
\begin{aligned}
\text { LHS of }(2-19) & \leqslant C \sum_{j^{\prime}} 2^{2 j^{\prime} s} \sum_{k=0}^{\infty} \sum_{\ell=1}^{d(k)}\left\|\chi\left(\frac{\rho}{2^{j^{\prime}}}\right)\left[\mathcal{H}_{\mu(k)} a_{k, \ell}\right](\rho) \rho^{\frac{n-1}{2}}\right\|_{L^{2}\left(\mathbb{R}^{+}\right)}^{2} \\
& =C \sum_{j^{\prime}} 2^{2 j^{\prime} s}\left\|\chi\left(\frac{\rho}{2^{j^{\prime}}}\right) \sum_{k=0}^{\infty} \sum_{\ell=1}^{d(k)} 2 \pi i^{k}\left[\mathscr{H}_{\mu(k)} a_{k, \ell}\right](\rho) Y_{k, \ell}(\omega)\right\|_{L^{2}\left(\mathbb{R}^{n}\right)}^{2} \\
& =C \sum_{j^{\prime}} 2^{2 j^{\prime} s}\left\|\chi\left(\frac{\rho}{2^{j^{\prime}}}\right) \hat{f}\right\|_{L^{2}\left(\mathbb{R}^{n}\right)}^{2} \sim\|f\|_{\dot{H}^{s}}^{2} .
\end{aligned}
$$

We can use a similar argument to prove

$$
\text { LHS of }(2-19) \geqslant c\|f\|_{\dot{H}^{s}}^{2} \text {. }
$$

This concludes the proof of Lemma 2.4.

\section{Proof of the main theorems}

In this section, we first use the spherical harmonic expansion to write the solution as a linear combination of products of the Hankel transform of radial functions and spherical harmonics. We prove the main theorems by analyzing a property of the Hankel transform. The key ingredients are to use the stationary phase argument and to exploit the asymptotic behavior of the Bessel function.

The expression of the solution. Consider the following Cauchy problem:

$$
\left\{\begin{array}{l}
i \partial_{t} u-\Delta u+\frac{a}{|x|^{2}} u=0, \\
u(x, 0)=u_{0}(x)
\end{array}\right.
$$

We use the spherical harmonic expansion to write

$$
u_{0}(x)=\sum_{k=0}^{\infty} \sum_{\ell=1}^{d(k)} a_{k, \ell}^{0}(r) Y_{k, \ell}(\theta) .
$$

Let us consider (3-1) in polar coordinates. Write $v(t, r, \theta)=u(t, r \theta)$ and $g(r, \theta)=$ $u_{0}(r \theta)$. Then $v(t, r, \theta)$ satisfies

$$
\left\{\begin{array}{l}
i \partial_{t} v-\partial_{r r} v-\frac{n-1}{r} \partial_{r} v-\frac{1}{r^{2}} \Delta_{\theta} v+\frac{a}{r^{2}} v=0, \\
v(0, r, \theta)=g(r, \theta) .
\end{array}\right.
$$

By (3-2), we have

$$
g(r, \theta)=\sum_{k=0}^{\infty} \sum_{\ell=1}^{d(k)} a_{k, \ell}^{0}(r) Y_{k, \ell}(\theta) .
$$


Using separation of variables, we can write $v$ as a linear combination of products of radial functions and spherical harmonics:

$$
v(t, r, \theta)=\sum_{k=0}^{\infty} \sum_{\ell=1}^{d(k)} v_{k, \ell}(t, r) Y_{k, \ell}(\theta),
$$

where $v_{k, \ell}$ is given by

$$
\left\{\begin{array}{l}
i \partial_{t} v_{k, \ell}-\partial_{r r} v_{k, \ell}-\frac{n-1}{r} \partial_{r} v_{k, \ell}+\frac{k(k+n-2)+a}{r^{2}} v_{k, \ell}=0, \\
v_{k, \ell}(0, r)=a_{k, \ell}^{0}(r)
\end{array}\right.
$$

for each $k, \ell \in \mathbb{N}, 1 \leqslant \ell \leqslant d(k)$. Then we can rewrite the above by the definition of $A_{v(k)}$ as

$$
\left\{\begin{array}{l}
i \partial_{t} v_{k, \ell}+A_{v(k)} v_{k, \ell}=0, \\
v_{k, \ell}(0, r)=a_{k, \ell}^{0}(r)
\end{array}\right.
$$

Applying the Hankel transform to (3-5), by Lemma 2.3(iv), we have

$$
\left\{\begin{array}{l}
i \partial_{t} \tilde{v}_{k, \ell}+\rho^{2} \tilde{v}_{k, \ell}=0 \\
\tilde{v}_{k, \ell}(0, \rho)=b_{k, \ell}^{0}(\rho)
\end{array}\right.
$$

where

$$
\tilde{v}_{k, \ell}(t, \rho)=\left(\mathscr{H}_{\nu} v_{k, \ell}\right)(t, \rho), \quad b_{k, \ell}^{0}(\rho)=\left(\mathscr{H}_{\nu} a_{k, \ell}^{0}\right)(\rho) .
$$

Solving this ODE and inverting the Hankel transform, we obtain

$$
\begin{aligned}
v_{k, \ell}(t, r) & =\int_{0}^{\infty}(r \rho)^{-\frac{n-2}{2}} J_{v(k)}(r \rho) \tilde{v}_{k, \ell}(t, \rho) \rho^{n-1} \mathrm{~d} \rho \\
& =\int_{0}^{\infty}(r \rho)^{-\frac{n-2}{2}} J_{v(k)}(r \rho) e^{i t \rho^{2}} b_{k, \ell}^{0}(\rho) \rho^{n-1} \mathrm{~d} \rho .
\end{aligned}
$$

Therefore we get

$$
\begin{aligned}
u(x, t) & =v(t, r, \theta) \\
& =\sum_{k=0}^{\infty} \sum_{\ell=1}^{d(k)} Y_{k, \ell}(\theta) \int_{0}^{\infty}(r \rho)^{-\frac{n-2}{2}} J_{v(k)}(r \rho) e^{i t \rho^{2}} b_{k, \ell}^{0}(\rho) \rho^{n-1} \mathrm{~d} \rho \\
& =\sum_{k=0}^{\infty} \sum_{\ell=1}^{d(k)} Y_{k, \ell}(\theta) \mathscr{H}_{v(k)}\left[e^{i t \rho^{2}} b_{k, \ell}^{0}(\rho)\right](r) .
\end{aligned}
$$


Proof of Theorem 1.1. By the Sobolev embedding $\dot{H}^{\frac{1}{2}-}(\mathbb{R}) \cap \dot{H}^{\frac{1}{2}+}(\mathbb{R}) \hookrightarrow L^{\infty}(\mathbb{R})$, it suffices to show:

Proposition 3.1. Let $\alpha \geqslant \frac{1}{2}-\frac{\beta}{4}$ and $\beta=1+$ be such that

$$
2 \alpha-1+\frac{\beta}{2}<1+\min \left\{\frac{n-2}{2},\left(\frac{(n-2)^{2}}{4}+a\right)^{\frac{1}{2}}\right\} \text {. }
$$

There exists a constant $C$ independent of $u_{0}$ such that

$$
\left.\int_{\mathbb{R}^{n}} \int_{\mathbb{R}}\left|\partial_{t}^{\alpha} u(x, t)\right|^{2} \frac{\mathrm{d} t \mathrm{~d} x}{(1+|x|)^{\beta}} \leqslant C\left\|u_{0}\right\|_{\dot{H}^{2 \alpha-1+\frac{\beta}{2}}}^{2} \mathbb{R}^{n}\right)
$$

Proof. By the Plancherel theorem with respect to time $t$, we obtain

$$
\text { LHS of (3-9) }=\int_{\mathbb{R}^{n}} \int_{\mathbb{R}}\left|\tau^{\alpha} \int_{\mathbb{R}} e^{-i t \tau} u(x, t) \mathrm{d} t\right|^{2} \frac{\mathrm{d} \tau \mathrm{d} x}{(1+|x|)^{\beta}} .
$$

Using (3-8), this is bounded above by

$$
\begin{aligned}
& \int_{\mathbb{R}^{n+1}}\left|\tau^{\alpha} \sum_{k=0}^{\infty} \sum_{\ell=1}^{d(k)} Y_{k, \ell}(\theta) \int_{\mathbb{R}} \int_{0}^{\infty}(r \rho)^{-\frac{n-2}{2}} J_{v(k)}(r \rho) e^{i t\left(\rho^{2}-\tau\right)} b_{k, \ell}^{0}(\rho) \rho^{n-1} \mathrm{~d} \rho \mathrm{d} t\right|^{2} \\
& \times \frac{\mathrm{d} \tau \mathrm{d} x}{(1+|x|)^{\beta}} \\
& \lesssim \int_{\mathbb{R}^{n+1}}\left|\tau^{\alpha} \sum_{k=0}^{\infty} \sum_{\ell=1}^{d(k)} Y_{k, \ell}(\theta) \int_{0}^{\infty}(r \rho)^{-\frac{n-2}{2}} J_{v(k)}(r \rho) b_{k, \ell}^{0}(\rho) \rho^{n-1} \delta\left(\tau-\rho^{2}\right) \mathrm{d} \rho\right|^{2} \\
& \times \frac{\mathrm{d} \tau \mathrm{d} x}{(1+|x|)^{\beta}} \\
& \lesssim \int_{\mathbb{R}^{n}} \int_{0}^{\infty}\left|\sum_{k=0}^{\infty} \sum_{\ell=1}^{d(k)} Y_{k, \ell}(\theta) \rho^{\alpha}(r \sqrt{\rho})^{-\frac{n-2}{2}} J_{\nu(k)}(r \sqrt{\rho}) b_{k, \ell}^{0}(\sqrt{\rho}) \rho^{\frac{n-1}{2}} \rho^{-\frac{1}{2}}\right|^{2} \\
& \times \frac{\mathrm{d} \rho \mathrm{d} x}{(1+|x|)^{\beta}} .
\end{aligned}
$$

By orthogonality, therefore, the LHS of (3-9) is

$$
\lesssim \sum_{k=0}^{\infty} \sum_{\ell=1}^{d(k)} \int_{0}^{\infty} \int_{0}^{\infty}\left|\rho^{2 \alpha+\frac{1}{2}}(r \rho)^{-\frac{n-2}{2}} J_{v(k)}(r \rho) b_{k, \ell}^{0}(\rho) \rho^{n-2}\right|^{2} \frac{\mathrm{d} \rho r^{n-1} \mathrm{~d} r}{(1+r)^{\beta}}
$$

Let $\chi$ be a smoothing function equals 1 in $\left[1, \frac{3}{2}\right]$ and vanishes outside $\left[\frac{1}{2}, 2\right]$. For our purpose, we make a dyadic decomposition to obtain 
LHS of (3-9)

$$
\begin{aligned}
& \lesssim \sum_{k=0}^{\infty} \sum_{\ell=1}^{d(k)} \sum_{M \in 2^{\mathbb{Z}}} \int_{0}^{\infty} \int_{0}^{\infty}\left|\rho^{2 \alpha+\frac{1}{2}}(r \rho)^{-\frac{n-2}{2}} J_{\nu(k)}(r \rho) b_{k, \ell}^{0}(\rho) \rho^{n-2} \chi\left(\frac{\rho}{M}\right)\right|^{2} \\
& \lesssim \sum_{k=0}^{\infty} \sum_{\ell=1}^{d(k)} \sum_{M \in 2^{\mathbb{Z}}} M^{2\left(n-2+2 \alpha+\frac{1}{2}\right)+1-n} \\
& \times \frac{r^{n-1} \mathrm{~d} r \mathrm{~d} \rho}{(1+r)^{\beta}} \\
& \times \int_{0}^{\infty} \int_{0}^{\infty}\left|(r \rho)^{-\frac{n-2}{2}} J_{v(k)}(r \rho) b_{k, \ell}^{0}(M \rho) \chi(\rho)\right|^{2} \frac{r^{n-1} \mathrm{~d} r \mathrm{~d} \rho}{\left(1+\frac{r}{M}\right)^{\beta}} \\
& \lesssim \sum_{k=0}^{\infty} \sum_{\ell=1}^{d(k)} \sum_{M \in 2^{\mathbb{Z}}} \sum_{R \in 2^{\mathbb{Z}}} \\
& M^{n-2+4 \alpha} R^{n-1} \\
& \times \int_{R}^{2 R} \int_{0}^{\infty}\left|(r \rho)^{-\frac{n-2}{2}} J_{v(k)}(r \rho) b_{k, \ell}^{0}(M \rho) \chi(\rho)\right|^{2} \frac{\mathrm{d} r \mathrm{~d} \rho}{\left(1+\frac{r}{M}\right)^{\beta}} .
\end{aligned}
$$

Define

$$
G_{k, \ell}(R, M)=\int_{R}^{2 R} \int_{0}^{\infty}\left|(r \rho)^{-\frac{n-2}{2}} J_{\nu(k)}(r \rho) b_{k, \ell}^{0}(M \rho) \chi(\rho)\right|^{2} \frac{\mathrm{d} r \mathrm{~d} \rho}{\left(1+\frac{r}{M}\right)^{\beta}} .
$$

Proposition 3.2. (1) If $R \lesssim 1$, then

$$
G_{k, \ell}(R, M) \lesssim R^{2 v(k)-n+3} M^{-n} \min \left\{1,\left(\frac{M}{R}\right)^{\beta}\right\}\left\|b_{k, \ell}^{0}(\rho) \chi\left(\frac{\rho}{M}\right) \rho^{\frac{n-1}{2}}\right\|_{L^{2}}^{2} .
$$

(2) If $R \gg 1$, then

$$
G_{k, \ell}(R, M) \lesssim \min \left\{1,\left(\frac{M}{R}\right)^{\beta}\right\} R^{-(n-2)} M^{-n}\left\|b_{k, \ell}^{0}(\rho) \chi\left(\frac{\rho}{M}\right) \rho^{\frac{n-1}{2}}\right\|_{L^{2}}^{2} .
$$

Proof. (1) Since $\rho \sim 1$, we have $r \rho \lesssim 1$. By the property (2-5) of the Bessel function, we obtain

$$
\begin{aligned}
G_{k, \ell}(R, M) & \lesssim \int_{R}^{2 R} \int_{0}^{\infty}\left|\frac{(r \rho)^{v(k)}(r \rho)^{-\frac{n-2}{2}}}{2^{v(k)} \Gamma\left(v(k)+\frac{1}{2}\right) \Gamma\left(\frac{1}{2}\right)} b_{k, \ell}^{0}(M \rho) \chi(\rho)\right|^{2} \mathrm{~d} \rho \frac{\mathrm{d} r}{\left(1+\frac{r}{M}\right)^{\beta}} \\
& \lesssim R^{2 v(k)-n+3} M^{-n} \min \left\{1,\left(\frac{M}{R}\right)^{\beta}\right\}\left\|b_{k, \ell}^{0}(\rho) \chi\left(\frac{\rho}{M}\right) \rho^{\frac{n-1}{2}}\right\|_{L^{2}}^{2} .
\end{aligned}
$$

(2) Since $\rho \sim 1$, we have $r \rho \gg 1$. We estimate

(3-10) $G_{k, \ell}(R, M)$

$$
\lesssim R^{-(n-2)} \int_{0}^{\infty}\left|b_{k, \ell}^{0}(M \rho) \chi(\rho)\right|^{2} \int_{R}^{2 R}\left|J_{v(k)}(r \rho)\right|^{2} \frac{\mathrm{d} r}{\left(1+\frac{r}{M}\right)^{\beta}} \mathrm{d} \rho .
$$

Subcase (i): $R \lesssim M$. Noting that $\rho \sim 1$, we obtain by Lemma 2.2 


$$
\int_{R}^{2 R}\left|J_{v(k)}(r \rho)\right|^{2} \frac{\mathrm{d} r}{\left(1+\frac{r}{M}\right)^{\beta}} \lesssim \int_{R}^{2 R}\left|J_{v(k)}(r \rho)\right|^{2} \mathrm{~d} r \lesssim 1 .
$$

Subcase (ii): $R \gg M$. Noticing that $\rho \sim 1$ again, we obtain by Lemma 2.2

$$
\int_{R}^{2 R}\left|J_{v(k)}(r \rho)\right|^{2} \frac{\mathrm{d} r}{\left(1+\frac{r}{M}\right)^{\beta}} \lesssim\left(\frac{M}{R}\right)^{\beta} \int_{R}^{2 R}\left|J_{v(k)}(r \rho)\right|^{2} \mathrm{~d} r \lesssim\left(\frac{M}{R}\right)^{\beta} .
$$

Putting (3-11) and (3-12) into (3-10), we have

$$
\begin{aligned}
G_{k, \ell}(R, M) & \lesssim \min \left\{1,\left(\frac{M}{R}\right)^{\beta}\right\} R^{-(n-2)} \int_{0}^{\infty}\left|b_{k, \ell}^{0}(M \rho) \chi(\rho)\right|^{2} \mathrm{~d} \rho \\
& \lesssim \min \left\{1,\left(\frac{M}{R}\right)^{\beta}\right\} R^{-(n-2)} M^{-n}\left\|b_{k, \ell}^{0}(\rho) \chi\left(\frac{\rho}{M}\right) \rho^{\frac{n-1}{2}}\right\|_{L^{2}}^{2} .
\end{aligned}
$$

Thus we have proved Proposition 3.2.

Now we return to proving Proposition 3.1. By Proposition 3.2, we show

$$
\begin{aligned}
& \int_{\mathbb{R}^{n}} \int_{\mathbb{R}}\left|\partial_{t}^{\alpha} u(x, t)\right|^{2} \frac{\mathrm{d} t \mathrm{~d} x}{(1+|x|)^{\beta}} \\
& \lesssim \sum_{k=0}^{\infty} \sum_{\ell=1}^{d(k)} \sum_{M \in 2^{\mathbb{Z}}} \sum_{\left\{R \in 2^{\mathbb{Z}}: R \lesssim 1\right\}}\left(M^{4 \alpha-2} R^{2(v(k)+1)} \min \left\{1,\left(\frac{M}{R}\right)^{\beta}\right\}\right. \\
& \left.\times\left\|b_{k, \ell}^{0}(\rho) \chi\left(\frac{\rho}{M}\right) \rho^{\frac{n-1}{2}}\right\|_{L^{2}}^{2}\right) \\
& +\sum_{k=0}^{\infty} \sum_{\ell=1}^{d(k)} \sum_{M \in 2^{\mathbb{Z}}\left\{R \in 2^{\mathbb{Z}}: R \gg 1\right\}} M^{4 \alpha-2+\beta} R^{1-\beta}\left\|b_{k, \ell}^{0}(\rho) \chi\left(\frac{\rho}{M}\right) \rho^{\frac{n-1}{2}}\right\|_{L^{2}}^{2} .
\end{aligned}
$$

From $\beta=1+$, one has

$$
\begin{aligned}
\sum_{M \in 2^{\mathbb{Z}}\left\{R \in 2^{\mathbb{Z}}: R \lesssim 1\right\}} M^{4 \alpha-2} R^{2(v(k)+1)} & \min \left\{1,\left(\frac{M}{R}\right)^{\beta}\right\}\left\|b_{k, \ell}^{0}(\rho) \chi\left(\frac{\rho}{M}\right) \rho^{\frac{n-1}{2}}\right\|_{L^{2}}^{2} \\
& \lesssim \sum_{M \in 2^{\mathbb{Z}}} M^{4 \alpha-2+\beta}\left\|b_{k, \ell}^{0}(\rho) \chi\left(\frac{\rho}{M}\right) \rho^{\frac{n-1}{2}}\right\|_{L^{2}}^{2} .
\end{aligned}
$$

Since $\alpha \geqslant \frac{1}{2}-\frac{\beta}{4}$, we have by Lemma 2.5

$$
\begin{aligned}
\int_{\mathbb{R}^{n}} \int_{\mathbb{R}}\left|\partial_{t}^{\alpha} u(x, t)\right|^{2} \frac{\mathrm{d} t \mathrm{~d} x}{(1+|x|)^{\beta}} & \lesssim \sum_{k=0}^{\infty} \sum_{\ell=1}^{d(k)} \sum_{M \in 2^{\mathbb{Z}}} M^{4 \alpha-2+\beta}\left\|b_{k, \ell}^{0}(\rho) \chi\left(\frac{\rho}{M}\right) \rho^{\frac{n-1}{2}}\right\|_{L^{2}}^{2} \\
& \leqslant C\left\|u_{0}\right\|_{\dot{H}^{2 \alpha-1+\frac{\beta}{2}}\left(\mathbb{R}^{n}\right)}^{2}
\end{aligned}
$$

Finally, we apply Proposition 3.1 with $\alpha=\frac{1}{2}+$ and $\alpha=\frac{1}{2}-$ to prove Theorem 1.1. 
Proof of Theorem 1.2. We now construct an example to show Theorem 1.2. The main idea is the stationary phase argument. By (3-8), we recall

$$
u(x, t)=\sum_{k=0}^{\infty} \sum_{\ell=1}^{d(k)} Y_{k, \ell}(\theta) \int_{0}^{\infty}(r \rho)^{-\frac{n-2}{2}} J_{v(k)}(r \rho) e^{i t \rho^{2}} b_{k, \ell}^{0}(\rho) \rho^{n-1} \mathrm{~d} \rho
$$

where

$$
b_{k, \ell}^{0}(\rho)=\left(\mathcal{H}_{\nu} a_{k, \ell}^{0}\right)(\rho), \quad u_{0}(x)=u_{0}(r \theta)=\sum_{k=0}^{\infty} \sum_{\ell=1}^{d(k)} a_{k, \ell}^{0}(r) Y_{k, \ell}(\theta) .
$$

In particular we choose $u_{0}(x)$ to be a radial function such that $\left(\mathscr{H}_{\nu(0)} u_{0}\right)(\xi)=$ $\chi_{N}(|\xi|)$, where $\chi_{N}$ is a smooth positive function supported in $J_{N}$ (to be chosen later) and $N \gg 1$. Then

$$
u(x, t)=\int_{0}^{\infty}(r \rho)^{-\frac{n-2}{2}} J_{v(0)}(r \rho) e^{i t \rho^{2}} \chi_{N}(\rho) \rho^{n-1} \mathrm{~d} \rho .
$$

Recalling the asymptotic expansion of the Bessel function,

$$
J_{\nu}(r)=r^{-\frac{1}{2}} \sqrt{\frac{2}{\pi}} \cos \left(r-\frac{\nu \pi}{2}-\frac{\pi}{4}\right)+O_{v}\left(r^{-\frac{3}{2}}\right) \quad \text { as } r \rightarrow \infty,
$$

with a constant depending on $v$ (see [Stein and Weiss 1971]), we can write

$$
\begin{array}{r}
u(x, t)=C_{v} \int_{0}^{\infty}(r \rho)^{-\frac{n-1}{2}}\left(e^{i\left(r \rho-\frac{v \pi}{2}-\frac{\pi}{4}\right)}-e^{-i\left(r \rho-\frac{v \pi}{2}-\frac{\pi}{4}\right)}\right) e^{i t \rho^{2}} \chi_{N}(\rho) \rho^{n-1} \mathrm{~d} \rho \\
+C_{v} \int_{0}^{\infty}(r \rho)^{-\frac{n-2}{2}} O_{v}\left((r \rho)^{-\frac{3}{2}}\right) e^{i t \rho^{2}} \chi_{N}(\rho) \rho^{n-1} \mathrm{~d} \rho .
\end{array}
$$

Let us define

$$
\begin{aligned}
& I_{1}(r)=C_{\nu} e^{i\left(\frac{v \pi}{2}+\frac{\pi}{4}\right)} \int_{0}^{\infty}(r \rho)^{-\frac{n-1}{2}} e^{i\left(-r \rho+t \rho^{2}\right)} \chi_{N}(\rho) \rho^{n-1} \mathrm{~d} \rho, \\
& I_{2}(r)=C_{\nu} e^{-i\left(\frac{v \pi}{2}+\frac{\pi}{4}\right)} \int_{0}^{\infty}(r \rho)^{-\frac{n-1}{2}} e^{i\left(r \rho+t \rho^{2}\right)} \chi_{N}(\rho) \rho^{n-1} \mathrm{~d} \rho, \\
& I_{3}(r)=C_{\nu} \int_{0}^{\infty}(r \rho)^{-\frac{n-2}{2}} O_{\nu}\left((r \rho)^{-\frac{3}{2}}\right) e^{i t \rho^{2}} \chi_{N}(\rho) \rho^{n-1} \mathrm{~d} \rho
\end{aligned}
$$

Let $\phi_{r}(\rho)=t \rho^{2}-r \rho$. The fundamental idea is to choose sets $J_{N}$ and $E \subset B^{n}$, in which $t(r)$ can be chosen, so that $\partial_{\rho} \phi_{r}(\rho)=2 t(r) \rho-r$ almost vanishes for all $\rho \in J_{N}$ and $r \in\{|x|: x \in E\}$. To this end, we choose

$$
E=\left\{x: \frac{1}{100} \leqslant|x| \leqslant \frac{1}{8}\right\} \quad \text { and } \quad J_{N}=\left[N, N+2 N^{\frac{1}{2}}\right] .
$$


Choose $t(r)=r /(2(N+\sqrt{N}))$; then $\partial_{\rho} \phi_{r}\left(N+N^{\frac{1}{2}}\right)=0$. Then

$$
\begin{aligned}
& I_{1}(r)=C_{\nu} e^{i\left(\frac{\nu \pi}{2}+\frac{\pi}{4}\right)} e^{i \phi_{r}(N+\sqrt{N})} \\
& \qquad \int_{0}^{\infty}(r \rho)^{-\frac{n-1}{2}} \exp \frac{i r[\rho-(N+\sqrt{N})]^{2}}{2(N+\sqrt{N})} \chi_{N}(\rho) \rho^{n-1} \mathrm{~d} \rho .
\end{aligned}
$$

Observe that

$$
\left|I_{1}(r)\right| \geqslant c_{\nu} \int_{0}^{\infty}(r \rho)^{-\frac{n-1}{2}} \cos \frac{r[\rho-(N+\sqrt{N})]^{2}}{2(N+\sqrt{N})} \chi_{N}(\rho) \rho^{n-1} \mathrm{~d} \rho .
$$

Moreover, there exists a small constant $c>0$ such that

$$
\cos \frac{r[\rho-(N+\sqrt{N})]^{2}}{2(N+\sqrt{N})} \geqslant c
$$

since $\frac{r[\rho-(N+\sqrt{N})]^{2}}{2(N+\sqrt{N})} \leqslant \frac{\pi}{4}$ for all $r \in\left[\frac{1}{100}, \frac{1}{8}\right], N \gg 1$ and $\rho \in J_{N}$. Therefore,

$$
\left|I_{1}(r)\right| \geqslant c_{\nu} r^{-\frac{n-1}{2}} \int_{0}^{\infty} \chi_{N}(\rho) \rho^{\frac{n-1}{2}} \mathrm{~d} \rho \geqslant c_{\nu} r^{-\frac{n-1}{2}} N^{\frac{n}{2}} .
$$

On the other hand, let $\varphi_{r}(\rho)=t \rho^{2}+r \rho, t=t(r)$ as before; then $\partial_{\rho} \varphi_{r}(\rho)=$ $2 t(r) \rho+r \geqslant \frac{1}{200}$ when $\rho \in J_{N}$ and $r \in\left[\frac{1}{100}, \frac{1}{8}\right]$. Integrating by parts, we obtain

$$
\left|I_{2}(r)\right| \leqslant C_{\nu} r^{-\frac{n}{2}} N^{\frac{n-2}{2}} \text {. }
$$

Obviously, we have

$$
\left|I_{3}(r)\right| \leqslant C_{\nu} r^{-\frac{n}{2}} N^{\frac{n-2}{2}} .
$$

Combining (3-19)-(3-21), we get for $N \gg 1$ and $r \in\left[\frac{1}{100}, \frac{1}{8}\right]$

$$
u^{*}(x) \geqslant c N^{\frac{n}{2}} \text {. }
$$

On the other hand, let $j_{0}=\log _{2} N$; we obtain by the definitions of $P_{j}$ and $\tilde{P}_{j}$

$$
\left\|u_{0}(x)\right\|_{H^{s}}^{2}=\sum_{j} 2^{2 j s}\left\|P_{j} u_{0}\right\|_{L^{2}}^{2}=\sum_{j} 2^{2 j s}\left\|P_{j} \tilde{P}_{j_{0}} u_{0}\right\|_{L^{2}}^{2} .
$$

By Lemma 2.4, we choose $s<\epsilon<1+\min \left\{\frac{n-2}{2},\left(\frac{(n-2)^{2}}{4}+a\right)^{\frac{1}{2}}\right\}$ to obtain

$$
\begin{aligned}
\left\|u_{0}(x)\right\|_{H^{s}}^{2} & \leqslant C \sum_{j} 2^{2 j s-2 \epsilon\left|j-j_{0}\right|}\left\|u_{0}\right\|_{L^{2}}^{2} \\
& =C N^{2 s} \sum_{j} 2^{2 j s-2 \epsilon|j|}\left\|\chi_{N}\right\|_{L^{2}}^{2}=N^{2 s+n-\frac{1}{2}} .
\end{aligned}
$$


Thus, by (1-5) and (3-22), we must have $s \geqslant \frac{1}{4}$.

Proof of Theorem 1.3. Even though there is a loss of the angular regularity in Theorem 1.3, the result implies the sharp result for the radial initial data. The key ingredient here is the following lemma proved in [Gigante and Soria 2008].

Lemma 3.1. Let $\tilde{J}_{v}(s)=s^{\frac{1}{2}} J_{v}(s)$ with $s \geqslant 0$, and let

$$
T_{\nu} g(r)=\int_{I} \frac{e^{i t(r) \rho^{2}} \tilde{J}_{\nu}(r \rho)}{\rho^{\frac{1}{4}}} g(\rho) \mathrm{d} \rho .
$$

Then

$$
\int_{0}^{1}\left|T_{\nu} g(r)\right|^{2} \mathrm{~d} r \leqslant C \int_{I}|g(\rho)|^{2} \mathrm{~d} \rho
$$

where the constant $C$ is independent of $g \in L^{2}(I)$, of the interval I, of the measurable function $t(r)$ and of the order $v \geqslant 0$.

We also can follow the Carleson approach [1980] to linearize our maximal operator, by making $t$ into a function of $r, t(r)$. By the triangle inequality, we have the estimate

$\left\|u^{*}(x)\right\|_{L^{2}\left(B^{n}\right)}$

$$
\leqslant C \sum_{k=0}^{\infty} \sum_{\ell=1}^{d(k)}\left\|\int_{0}^{\infty}(r \rho)^{-\frac{n-2}{2}} J_{v(k)}(r \rho) e^{i t(r) \rho^{2}} b_{k, \ell}^{0}(\rho) \rho^{n-1} \mathrm{~d} \rho\right\|_{L_{r n-1}^{2} \mathrm{~d} r} .
$$

Let $g(\rho)=b_{k, \ell}^{0}(\rho) \rho^{\frac{n-1}{2}+\frac{1}{4}} ;$ then

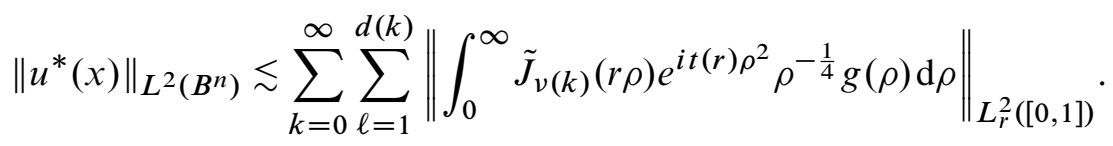

Using Lemma 3.1, we obtain

$$
\left\|u^{*}(x)\right\|_{L^{2}\left(B^{n}\right)} \lesssim C \sum_{k=0}^{\infty} \sum_{\ell=1}^{d(k)}\left\|b_{k, \ell}^{0}(\rho) \rho^{\frac{n-1}{2}+\frac{1}{4}}\right\|_{L_{\rho}^{2}\left(\mathbb{R}^{+}\right)} .
$$

Let $\alpha=(n-1) / 2+\epsilon$ with $\epsilon>0$, we have by the Cauchy-Schwarz inequality $\left\|u^{*}(x)\right\|_{L^{2}\left(B^{n}\right)}$

$$
\leqslant C\left(\sum_{k=0}^{\infty} \sum_{\ell=1}^{d(k)}(1+k)^{-2 \alpha}\right)^{\frac{1}{2}}\left(\sum_{k=0}^{\infty} \sum_{\ell=1}^{d(k)}(1+k)^{2 \alpha}\left\|b_{k, \ell}^{0}(\rho) \rho^{\frac{n-1}{2}+\frac{1}{4}}\right\|_{L_{\rho}^{2}\left(\mathbb{R}^{+}\right)}^{2}\right)^{\frac{1}{2}} .
$$


Since $d(k) \simeq\langle k\rangle^{n-2}$, we have by Lemma 2.5

$\left\|u^{*}(x)\right\|_{L^{2}\left(B^{n}\right)} \lesssim\left(\sum_{k=0}^{\infty} \sum_{\ell=1}^{d(k)}(1+k)^{2 \alpha}\left\|b_{k, \ell}^{0}(\rho) \rho^{\frac{n-1}{2}+\frac{1}{4}}\right\|_{L_{\rho}^{2}\left(\mathbb{R}^{+}\right)}^{2}\right)^{\frac{1}{2}} \lesssim\left\|u_{0}\right\|_{H_{r}{ }^{\frac{1}{4}} H_{\theta}^{\alpha}}$.

This completes the proof of Theorem 1.3.

\section{Acknowledgments}

The authors thank the referee and the associated editor for their invaluable comments and suggestions which helped improve the paper greatly. This work was supported in part by the NSF of China under grants 11171033, 11231006, and 11371059. The second author was partly supported by the Fundamental Research Foundation of BIT (20111742015) and RFDP (20121101120044), Beijing Natural Science Foundation (1144014), and National Natural Science Foundation of China (11401024). C. Miao was also supported by Beijing Center for Mathematics and Information Interdisciplinary Sciences.

\section{References}

[Bourgain 1995] J. Bourgain, "Some new estimates on oscillatory integrals", pp. 83-112 in Essays on Fourier analysis in honor of Elias M. Stein (Princeton, NJ, 1991), edited by C. Fefferman et al., Princeton Math. Ser. 42, Princeton University Press, 1995. MR 96c:42028 Zbl 0840.42007

[Bourgain 2013] J. Bourgain, "On the Schrödinger maximal function in higher dimension", Proc. Steklov Inst. Math. 280 (2013), 46-60. Zbl 1291.35253

[Burq et al. 2003] N. Burq, F. Planchon, J. G. Stalker, and A. S. Tahvildar-Zadeh, "Strichartz estimates for the wave and Schrödinger equations with the inverse-square potential", J. Funct. Anal. 203:2 (2003), 519-549. MR 2004m:35025 Zbl 1030.35024

[Burq et al. 2004] N. Burq, F. Planchon, J. G. Stalker, and A. S. Tahvildar-Zadeh, "Strichartz estimates for the wave and Schrödinger equations with potentials of critical decay", Indiana Univ. Math. J. 53:6 (2004), 1665-1680. MR 2005k:35241 Zbl 1084.35014

[Carleson 1980] L. Carleson, "Some analytic problems related to statistical mechanics", pp. 5-45 in Euclidean harmonic analysis (College Park, MD, 1979), edited by J. J. Benedetto, Lecture Notes in Math. 779, Springer, Berlin, 1980. MR 82j:82005 Zbl 0425.60091

[Cheeger and Taylor 1982] J. Cheeger and M. Taylor, "On the diffraction of waves by conical singularities, I”, Comm. Pure Appl. Math. 35:3 (1982), 275-331. MR 84h:35091a Zbl 0526.58049

[Cho et al. 2006] Y. Cho, S. Lee, and Y. Shim, "A maximal inequality associated to Schrödinger type equation”, Hokkaido Math. J. 35:4 (2006), 767-778. MR 2007m:42016 Zbl 1122.42008

[Dahlberg and Kenig 1982] B. E. J. Dahlberg and C. E. Kenig, "A note on the almost everywhere behavior of solutions to the Schrödinger equation", pp. 205-209 in Harmonic analysis (Minneapolis, MN, 1981), edited by F. Ricci and G. Weiss, Lecture Notes in Math. 908, Springer, Berlin, 1982. MR 83f:35023 Zbl 0519.35022

[Gigante and Soria 2008] G. Gigante and F. Soria, "On the boundedness in $H^{1 / 4}$ of the maximal square function associated with the Schrödinger equation”, J. Lond. Math. Soc. (2) 77:1 (2008), 51-68. MR 2010a:42039 Zbl 1218.42005 
[Kalf et al. 1975] H. Kalf, U.-W. Schmincke, J. Walter, and R. Wüst, "On the spectral theory of Schrödinger and Dirac operators with strongly singular potentials", pp. 182-226 in Spectral theory and differential equations (Dundee, 1974), edited by W. N. Everitt, Lecture Notes in Math. 448, Springer, Berlin, 1975. MR 53 \#1051 Zbl 0311.47021

[Lee 2006] S. Lee, "On pointwise convergence of the solutions to Schrödinger equations in $\mathbb{R}^{2}$ ”, Int. Math. Res. Not. 2006 (2006), Article ID \#32597. MR 2007j:35180 Zbl 1131.35306

[Machihara et al. 2005] S. Machihara, M. Nakamura, K. Nakanishi, and T. Ozawa, "Endpoint Strichartz estimates and global solutions for the nonlinear Dirac equation", J. Funct. Anal. 219:1 (2005), 1-20. MR 2006b:35199 Zbl 1060.35025

[Miao et al. 2013] C. Miao, J. Zhang, and J. Zheng, "Strichartz estimates for wave equation with inverse square potential”, Comm. Contemp. Math. 15:6 (2013), Article ID \#1350026. MR 3139408 Zbl 1284.35357

[Moyua et al. 1996] A. Moyua, A. Vargas, and L. Vega, "Schrödinger maximal function and restriction properties of the Fourier transform", Int. Math. Res. Not. 1996:16 (1996), 793-815. MR 97k:42042 Zbl 0868.35024

[Planchon et al. 2003a] F. Planchon, J. G. Stalker, and A. S. Tahvildar-Zadeh, "Dispersive estimate for the wave equation with the inverse-square potential", Discrete Contin. Dyn. Syst. 9:6 (2003), 1387-1400. MR 2005h:35212 Zbl 1047.35081

[Planchon et al. 2003b] F. Planchon, J. G. Stalker, and A. S. Tahvildar-Zadeh, " $L$ p estimates for the wave equation with the inverse-square potential", Discrete Contin. Dyn. Syst. 9:2 (2003), 427-442. MR 2003j:35195 Zbl 1031.35092

[Shao 2010] S. Shao, "On localization of the Schrödinger maximal operator", preprint, 2010. arXiv $1006.2787 \mathrm{v} 1$

[Sjölin 1987] P. Sjölin, "Regularity of solutions to the Schrödinger equation", Duke Math. J. 55:3 (1987), 699-715. MR 88j:35026 Zbl 0631.42010

[Stein 1993] E. M. Stein, Harmonic analysis: real-variable methods, orthogonality, and oscillatory integrals, Princeton Mathematical Series 43, Princeton University Press, 1993. MR 95c:42002 Zbl 0821.42001

[Stein and Weiss 1971] E. M. Stein and G. Weiss, Introduction to Fourier analysis on Euclidean spaces, Princeton Mathematical Series 32, Princeton University Press, 1971. MR 46 \#4102 Zbl 0232.42007

[Stempak 2000] K. Stempak, "A weighted uniform $L^{p}$-estimate of Bessel functions: a note on a paper of Guo", Proc. Amer. Math. Soc. 128:10 (2000), 2943-2945. MR 2000m:33004 Zbl 0951.33004

[Tao 2003] T. Tao, "A sharp bilinear restriction estimate for paraboloids", Geom. Funct. Anal. 13:6 (2003), 1359-1384. MR 2004m:47111 Zbl 1068.42011

[Tao and Vargas 2000] T. Tao and A. Vargas, "A bilinear approach to cone multipliers, II: Applications”, Geom. Funct. Anal. 10:1 (2000), 216-258. MR 2002e:42013 Zbl 0949.42013

[Vazquez and Zuazua 2000] J. L. Vazquez and E. Zuazua, "The Hardy inequality and the asymptotic behaviour of the heat equation with an inverse-square potential", J. Funct. Anal. 173:1 (2000), 103-153. MR 2001j:35122 Zbl 0953.35053

[Vega 1988] L. Vega, "Schrödinger equations: pointwise convergence to the initial data", Proc. Amer. Math. Soc. 102:4 (1988), 874-878. MR 89d:35046 Zbl 0654.42014

Received May 23, 2013. Revised January 1, 2014. 
ChAngXing MiaO

Institute of Applied Physics and Computational Mathematics

P. O. Box 8009

BEIJING, 100088

CHINA

miao_changxing@iapcm.ac.cn

JUNYONG ZHANG

DEPARTMENT OF MATHEMATICS

Beijing Institute of Technology

BEIJING, 100081

CHINA

zhang_junyong@bit.edu.cn

JIQIANG ZHENG

The Graduate School of China ACAdemy of Engineering Physics

P. O. BOX 2101

BEIJING, 100088

CHINA

zhengjiqiang@gmail.com 


\title{
PACIFIC JOURNAL OF MATHEMATICS
}

\author{
msp.org/pjm
}

Founded in 1951 by E. F. Beckenbach (1906-1982) and F. Wolf (1904-1989)

\section{EDITORS}

Don Blasius (Managing Editor)

Department of Mathematics

University of California

Los Angeles, CA 90095-1555

blasius@math.ucla.edu

\author{
Paul Balmer \\ Department of Mathematics \\ University of California \\ Los Angeles, CA 90095-1555 \\ balmer@math.ucla.edu \\ Robert Finn \\ Department of Mathematics \\ Stanford University \\ Stanford, CA 94305-2125 \\ finn@math.stanford.edu \\ Sorin Popa \\ Department of Mathematics \\ University of California \\ Los Angeles, CA 90095-1555 \\ popa@math.ucla.edu
}

\author{
Vyjayanthi Chari \\ Department of Mathematics \\ University of California \\ Riverside, CA 92521-0135 \\ chari@math.ucr.edu \\ Kefeng Liu \\ Department of Mathematics \\ University of California \\ Los Angeles, CA 90095-1555 \\ liu@math.ucla.edu \\ Jie Qing \\ Department of Mathematics \\ University of California \\ Santa Cruz, CA 95064 \\ qing@ cats.ucsc.edu
}

\section{PRODUCTION}

Silvio Levy, Scientific Editor, production@msp.org

\section{SUPPORTING INSTITUTIONS}

ACADEMIA SINICA, TAIPEI

CALIFORNIA INST. OF TECHNOLOGY

INST. DE MATEMÁTICA PURA E APLICADA

KEIO UNIVERSITY

MATH. SCIENCES RESEARCH INSTITUTE

NEW MEXICO STATE UNIV.

OREGON STATE UNIV.

\author{
STANFORD UNIVERSITY \\ UNIV. OF BRITISH COLUMBIA \\ UNIV. OF CALIFORNIA, BERKELEY \\ UNIV. OF CALIFORNIA, DAVIS \\ UNIV. OF CALIFORNIA, LOS ANGELES \\ UNIV. OF CALIFORNIA, RIVERSIDE \\ UNIV. OF CALIFORNIA, SAN DIEGO \\ UNIV. OF CALIF., SANTA BARBARA
}

\author{
Daryl Cooper \\ Department of Mathematics \\ University of California \\ Santa Barbara, CA 93106-3080 \\ cooper@math.ucsb.edu \\ Jiang-Hua Lu \\ Department of Mathematics \\ The University of Hong Kong \\ Pokfulam Rd., Hong Kong \\ jhlu@maths.hku.hk \\ Paul Yang \\ Department of Mathematics \\ Princeton University \\ Princeton NJ 08544-1000 \\ yang@math.princeton.edu
}

These supporting institutions contribute to the cost of publication of this Journal, but they are not owners or publishers and have no responsibility for its contents or policies.

See inside back cover or msp.org/pjm for submission instructions.

The subscription price for 2015 is US \$420/year for the electronic version, and \$570/year for print and electronic.

Subscriptions, requests for back issues and changes of subscribers address should be sent to Pacific Journal of Mathematics, P.O. Box 4163, Berkeley, CA 94704-0163, U.S.A. The Pacific Journal of Mathematics is indexed by Mathematical Reviews, Zentralblatt MATH, PASCAL CNRS Index, Referativnyi Zhurnal, Current Mathematical Publications and Web of Knowledge (Science Citation Index).

The Pacific Journal of Mathematics (ISSN 0030-8730) at the University of California, c/o Department of Mathematics, 798 Evans Hall \#3840, Berkeley, CA 94720-3840, is published twelve times a year. Periodical rate postage paid at Berkeley, CA 94704, and additional mailing offices. POSTMASTER: send address changes to Pacific Journal of Mathematics, P.O. Box 4163, Berkeley, CA 94704-0163.

PJM peer review and production are managed by EditFLOW ${ }^{\circledR}$ from Mathematical Sciences Publishers.

\section{PUBLISHED BY}

\section{mathematical sciences publishers \\ nonprofit scientific publishing}

http://msp.org/

(C) 2015 Mathematical Sciences Publishers 


\section{PACIFIC JOURNAL OF MATHEMATICS}

Volume $273 \quad$ No. $1 \quad$ January 2015

Maximal estimates for Schrödinger equations with inverse-square potential 1

ChangXing MiaO, JUNYong Zhang and JiQiang Zheng

Vassiliev Invariants of Virtual Legendrian Knots

PATRICIA CAHN and ASA LEVI

Some results on the generic vanishing of Koszul cohomology via

deformation theory

JIE WANG

Conformal metrics with constant curvature one and finitely many conical

singularities on compact Riemann surfaces

QING Chen, WeI WANG, Yingyi Wu and BIN XU

$\mathbb{Q}$-bases of the Néron-Severi groups of certain elliptic surfaces

MASAMICHI KURODA

On a prime zeta function of a graph

TAKehiro Hasegawa and SEIKEN SAIto

On Whittaker modules for a Lie algebra arising from the 2-dimensional torus 147

SHaObin TAN, QING WANG and ChENGKANG XU

Fréchet quantum supergroups

AXEL DE GOURSAC

Generators of the Gauss-Picard modular group in three complex dimensions

BaoHua Xie, JieYan Wang and YuePing Jiang

Complete characterization of isolated homogeneous hypersurface singularities

STEPHEN YAu and HuAiQING ZuO

A theorem of Mœglin and Waldspurger for covering groups

SHIV PRAKASH PATEL

Spanning trees and random walks on weighted graphs 